\title{
IL-17 and IL-11 GCF Levels in Aggressive and Chronic Periodontitis Patients: Relation to PCR Bacterial Detection
}

\author{
Olfat G. Shaker' ${ }^{1}$ and Noha A. Ghallab ${ }^{2}$ \\ ${ }^{1}$ Department of Medical Biochemistry \& Molecular Biology, Faculty of Medicine, Cairo University, Egypt \\ ${ }^{2}$ Department of Oral Medicine, Periodontology and Diagnosis, Faculty of Oral and Dental Medicine, \\ Cairo University, Egypt
}

Correspondence should be addressed to Olfat G. Shaker, olfatshaker@yahoo.com

Received 24 July 2012; Revised 28 September 2012; Accepted 15 October 2012

Academic Editor: Hidde Bult

Copyright ( 2012 O. G. Shaker and N. A. Ghallab. This is an open access article distributed under the Creative Commons Attribution License, which permits unrestricted use, distribution, and reproduction in any medium, provided the original work is properly cited.

\begin{abstract}
Objectives. This study evaluated IL-17 and IL-11 in gingival crevicular fluid (GCF) of generalized chronic periodontitis (GCP) and generalized aggressive periodontitis (GAgP) patients in relation to periodontopathic bacteria. Subjects and Methods. GCF samples were collected from 65 subjects including 25 CP, 25 GAgP, and 15 controls (C) and analyzed for IL-17 and IL-11 by an enzymelinked immunosorbent assay. Molecular detection of bacteria in the dental plaque was determined by polymerase chain reaction. Results. The total amount of IL-17 was significantly higher in GAgP group than in GCP and C groups $(P<0.001)$. The IL-11 concentration was significantly higher in C and GCP groups than GAgP group $(P<0.001)$. The IL-11/IL-17 ratio was significantly higher in the $\mathrm{C}$ group than in GCP and GAgP groups $(P<0.05)$. Moreover, GAgP group showed lower ratios of IL-11/IL-17 when compared to GCP group. The high positivity of $P$. gingivalis in the dental plaque was associated with significantly increased GCF levels of IL-17 in GCP and GAgP patients. Conclusions. The increased IL-17 level in GCF of GAgP suggests a potential role in the aetiopathogenesis. Meanwhile, the decreased ratio of IL-11/IL-17 might reflect an imbalance between the proinflammatory and anti-inflammatory cytokines in different periodontal diseases.
\end{abstract}

\section{Introduction}

Periodontitis is a chronic inflammatory disease characterized by destruction of tooth-supporting tissues [1]. The redcomplex bacteria including, Porphyromonas gingivalis, Tannerella forsythia and Treponema denticola were shown to have the closest association with the severity of periodontitis $[2,3]$ besides this group, Aggregatibacter actinomycetemcomitans and Prevotella intermedia. A complex cytokine network is synthesized in response to these periodontopathogenic bacteria [4] and plays an important role in periodontal disease [5]. The structure of bioactive bacterial compounds differs substantially between different bacteria and subsequently resulting in differences in cytokine production [6].

Exciting new evidence has emerged introducing a novel subset of T-helper (Th) lymphocytes termed "Th17" [7]. The role of Th17 cells and their specific cytokines (IL-17) in periodontal disease is just beginning to be investigated [8]. IL-17 is a proinflammatory cytokine that stimulates a variety of cells to produce inflammatory mediators such as IL-1, IL-6, and TNF- $\alpha$ [9]. Although Th17 pathways are mostly associated with protection against bacteria via recruitment of phagocytes [10], they have also been proposed to enhance alveolar bone resorption [11]. Previous studies showed that IL-17 was associated with chronic periodontitis [11-13] suggesting that it may contribute to periodontal tissue destruction.

IL-11 as an anti-inflammatory mediator has been shown to play an important role in the modulation of immune response via reduction of proinflammatory cytokine production in animal models [14]. It was predicted that IL-11 may be considered as a candidate molecule for therapeutic modulation of the host response in the management of periodontal diseases $[5,15,16]$. 
Regardless of the different clinical profiles between generalized aggressive periodontitis $(\mathrm{GAgP})$ and generalized chronic periodontitis (GCP), it is not clear whether those patients present a distinct profile of inflammatory mediators [17]. Based on the above, the aim of this study was to determine the total amount and concentration of the cytokines IL-17 and IL-11 in the gingival crevicular fluid (GCF) from GCP, GAgP patients, and healthy controls (C) and to investigate these levels in relation to the periodontal pathogens presented in their dental plaque. To date this is the first study to examine the GCF IL-17 and IL11 levels in GAgP and GCP patients in connection with periodontopathic bacteria in the dental plaque.

\section{Materials and Methods}

2.1. Study Population. Sixty-five systemically healthy subjects were selected from the Outpatient Clinic, Department of Oral Medicine and Periodontology, Faculty of Oral and Dental Medicine, Cairo University, between February 2010 and December 2010, who signed an informed consent form approved by the University Institutional Review Board after explaining the study. Exclusion criteria were as follows: pregnant women, subjects with $<22$ permanent teeth, having any given systemic disease, taking any type of medication and/or antibiotic therapy during the 3 months before the study, receiving previous periodontal treatment, and former or current smokers.

2.2. Studied Groups. Subjects with GAgP and GCP and healthy control (C) subjects were diagnosed based on the periodontal classification of The American Academy of Periodontology [1] and met the following criteria [18].

(i) GCP group $(n=25)$ : GCP patients were $>35$ years of age and had a minimum of six teeth with at least one site each with pocket depth (PD) and clinical attachment level (CAL) $>5 \mathrm{~mm}$.

(ii) GAgP group $(n=25)$ : GAgP patients were $<35$ years of age and had a minimum of six teeth other than the first molars and incisors with at least one site each with $\mathrm{PD}$ and $\mathrm{CAL}>5 \mathrm{~mm}$, and familial aggregation (subjects were asked if they had at least one other member of the family presenting or with a history of periodontal diseases).

(iii) Control group $(n=15)$ : C subjects were $>20$ years of age and had clinically healthy gingiva with zero plaque index, gingival index, and CAL $\leq 3 \mathrm{~mm}$ PD.

2.3. Clinical Parameters. All subjects received clinical examination including the following periodontal parameters: plaque index (PI), gingival index (GI), PD and CAL. One examiner performed all the measurements at six sites for all teeth mesiobuccal, mesiolingual, midbuccal, distobuccal, distolingual, and midlingual. Calibration exercises for probing measurements were performed in five patients before the actual study. The intraexaminer agreement was good, with a $0.84 \kappa$ value. PI was established by measuring the presence or absence of supragingival biofilm with a sweeping movement of the probe around the buccal, mesial, distal, and lingual regions of all teeth [19]. Marginal gingival bleeding was recorded with GI. PD was measured from the free-gingival margin to the base of the periodontal pocket and CAL was measured from the cementoenamel junction of the tooth to the base of the periodontal pocket. Measurements were rounded to the highest whole millimeter using the Michigan 0 probe with Williams' markings.

\subsection{Dental Plaque and GCF Samples Collection. Following} the careful removal of supragingival biofilm, areas were washed with water spray, isolated with cotton rolls, and gently dried. Subgingival biofilm samples were collected by sterile endodontic paper points (no. 35), which were inserted in the site with deepest periodontal pocket in each quarter and kept there for $30 \mathrm{~s}$. The paper points were placed into sterile tubes containing $300 \mu \mathrm{L}$ PBS [20]. GCF was collected from the same sites of microbiological sampling. After subgingival biofilm collection, teeth were washed again, and the area was isolated and gently dried. GCF was collected by placing filter paper strips (Perio-paper, IDE Interstate, Amityville, NY, USA) into the pocket until a slight resistance was perceived and then left in place for $30 \mathrm{~s}$. Strips contaminated by blood were excluded. Immediately, the volume of the sample was measured with the aid of a calibrated Periotron 800 (Oraflow Inc., Amityville, NY, USA). After volume measurements, the strips were placed into sterile eppendorf tubes containing $300 \mu \mathrm{L}$ PBS. All samples (subgingival biofilm and GCF) were immediately stored at $-20^{\circ} \mathrm{C}$ until subsequent analysis. Only one examiner, the same one charged with clinical measures, collected all microbial and GCF samples.

2.5. Determination of $I L-17$ and $I L-11$ in GCF Samples. GCF samples were analyzed for IL-17 and IL-11 using commercially available human enzyme-linked immunosorbent assay (ELISA) kits (BioSource Europe SA, Nivelles, Belgium, and PromoCell, Heidelberg, Germany, resp.). Analyses were performed according to the manufacturer's protocol. All ELISA determinations were performed in duplicate. It is a sandwich-type ELISA where a monoclonal anti-human IL17 and IL-11, adsorbed onto microwells, bind IL-17 and IL-11 in the sample, respectively. Results were calculated using the standard curves included in each assay kit. The intensity of the color was measured at $450 \mathrm{~nm}$. The total amount of IL-17 and IL-11 was determined in picograms (pg) and calculations of the concentration in each sample were performed by dividing the total amounts of IL-17 by the volume of the sample $(\mathrm{pg} / \mu \mathrm{L})$.

2.6. PCR for Dental Plaque Bacterial Detection. DNA was extracted from microbial samples using the DNA extraction kit (Roche, Mannheim, Germany) according to the manufacturer's recommendations. The conventional polymerase chain reaction (PCR) amplification of the conserved region of $16 \mathrm{~S}$ ribosomal DNA was tested for periodontal pathogens 
TABLE 1: Sequence of used primers and their PCR product size (bp).

\begin{tabular}{|c|c|c|}
\hline Primers & Sequences & PCR product size (bp) \\
\hline \multirow{2}{*}{ A. $a$} & 5'-GCTAATACCGCGTAGAGTCGG-3' & \multirow{2}{*}{443} \\
\hline & 5'-ATTTCACACCTCACTTAAAGGT-3' & \\
\hline \multirow{2}{*}{ P. $i$} & 5'-TTTGTTGGGGAGTAAAGCGGG-3' & \multirow{2}{*}{575} \\
\hline & 5' TCAACATCTCTGTATCCTGCGT-3' & \\
\hline \multirow{2}{*}{ P. $g$} & 5'-AGGCAGCTTGCCATACTGCG-3' & \multirow{2}{*}{404} \\
\hline & 5'-ACTGTTAGCAACTACCGATGT-3' & \\
\hline \multirow{2}{*}{ T. $f$} & 5'-GCGTATGTAACCTGCCCGCA-3' & \multirow{2}{*}{641} \\
\hline & 5'-TGCTTCAGTGTCAGTTATACCT-3' & \\
\hline \multirow{2}{*}{ T. $d$} & 5' -TAATACCGAATGTGCTCATTTACAT-3' & \multirow{2}{*}{316} \\
\hline & 5' -TCAAAGAAGCATTCССТСТTCTTCTTA-3' & \\
\hline
\end{tabular}

TABle 2: Demographic and clinical characteristics of study groups.

\begin{tabular}{lcccc}
\hline & GCP $(n=25)$ & GAgP $(n=25)$ & C $(n=15)$ & Mann-Whitney $U$ test \\
\hline Mean age, years & $40.2 \pm 2.65^{* \#}$ & $27.53 \pm 3.73$ & $25 \pm 2.58$ & $<0.001$ \\
Gender, M/F & $13 / 12$ & $11 / 14$ & $7 / 8$ & $<0.001$ \\
PI & $2.4 \pm 0.5^{* \#}$ & $1.8 \pm 0.9^{*}$ & $0 \pm 0$ & $<0.001$ \\
GI & $2.6 \pm 0.5^{* \#}$ & $1.7 \pm 0.8^{*}$ & $0 \pm 0$ & $<0.001$ \\
PPD, mm & $5.7 \pm 0.9^{*}$ & $6 \pm 1.4^{*}$ & $1.5 \pm 0.5$ & $<0.001$ \\
CAL, mm & $4.9 \pm 0.5^{* \#}$ & $7.2 \pm 1.1^{*}$ & $0 \pm 0$ & $<$ \\
\hline
\end{tabular}

Data represented as mean \pm SD. PI: plaque index; GI: gingival index; PPD: probing pocket depth; CAL: clinical attachment level.

GCP: generalized chronic periodontitis; GAgP: generalized aggressive periodontitis; C: control.

* Statistically significantly different from the C group.

\#Statistically significantly different from the GAgP group.

including A. actinomycetemcomitans, P. intermedia, P. gingivalis, T. forsythia, and T. denticola (Table 1). All these PCR primers were obtained commercially (Gibco BRL, São Paulo, SP, Brazil). $100 \mathrm{ng}$ of genomic DNA was added to the PCR mixture which contained $1 \mu \mathrm{mol} / \mathrm{L}$ of the primers, $2.5 \mathrm{U}$ of Taq polymerase in $1 \mathrm{x}$ buffer, and $0.2 \mathrm{mMol} / \mathrm{L}$ of $\mathrm{dCTP}$, dGTP, dATP, and dTTP in a total volume of $50 \mu \mathrm{L}$. PCR amplification was performed for 35 cycles of $30 \mathrm{~s}$ at $95^{\circ} \mathrm{C}, 30 \mathrm{~s}$ at $55^{\circ} \mathrm{C}$, and $60 \mathrm{~s}$ at $72^{\circ} \mathrm{C}$ in thermocycler. Twenty $\mu \mathrm{L}$ of each PCR reaction mixture was electrophoresed in $1.7 \%$ agarose gel in TAE buffer, and the amplification products were visualized under ultraviolet light, on ethidium bromidestained gel.

2.7. Statistical Analysis. Numerical data were presented as mean and standard deviation (SD) values. Qualitative data were presented as frequencies and percentages. One-way ANOVA (Analysis of Variance) was used to compare mean $\mathrm{PD}$ and CAL in the three groups. Tukey's test for pair-wise comparisons was used to determine significant differences between groups when ANOVA test was significant. KruskallWallis test was used to compare cytokine levels, PI, and GI in the three groups. Mann-Whitney $U$ test was used in the procedure of pair-wise comparisons when Kruskall-Wallis test was significant and was also used to compare cytokine levels in positive and negative bacteriological cases. Chisquare $\left(x^{2}\right)$ test was used to compare prevalence of different bacterial species in the three groups. The significance level was set at $P \leq 0.05$. Statistical analysis was performed with SPSS 18.0, Chicago, IL, USA.

\section{Results}

3.1. The Clinical Characteristics of the Patients. The periodontal values of the studied groups were shown in Table 2. All of the clinical parameters were significantly higher in GAgP and GCP groups compared to C group. PI, GI, and CAL values were significantly higher in GAgP group than GCP group.

3.2. Total Amount and Concentrations of IL-17 and IL-11 in GCF. The GCF volume, total amounts, and concentrations of IL-17 and IL-11 are shown in Table 3. The GCF volume was found to be the highest in GAgP group (significantly higher than GCP and $C$ groups, $P<0.001$ ), followed by GCP and $\mathrm{C}$ groups, respectively.

The total amount of IL-17 was significantly higher in GAgP group than in GCP and $\mathrm{C}$ groups $(P<0.001)$. The total amount of IL-17 was higher in GCP group than in C group but without statistical significance. Meanwhile, GAgP group had significantly lower IL-17 concentrations than GCP and $\mathrm{C}$ groups $(P<0.001)$. The difference between the GCP and $\mathrm{C}$ groups was also found to be significantly $(P<0.001)$ higher in $\mathrm{C}$ group $(P<0.001)$. The concentration of IL11 was significantly higher in $\mathrm{C}$ and GCP groups than in GAgP group $(P<0.001)$. The GAgP group had significantly 
TABLE 3: The gingival crevicular fluid (GCF) total amount (pg) and concentrations $(\mathrm{pg} / \mu \mathrm{L})$ of the cytokines IL-11 and IL-17 (mean \pm SD (minimum-maximum)) and GCF volume $(\mu \mathrm{L})$.

\begin{tabular}{lccrc}
\hline & GCP & Groups & C & P value from Kruskall-Wallis test \\
\hline IL-11 $(\mathrm{pg})$ & $53.1 \pm 20.9(29-112)$ & $56.5 \pm 20.1(16-80)$ & $49.9 \pm 28.8(14-87)$ & 0.309 \\
IL-11 $(\mathrm{pg} / \mu \mathrm{L})$ & $52.1 \pm 29.2(22-115)^{* \#}$ & $40.5 \pm 19.5(31-102)^{* \#}$ & $106.5 \pm 41.5(56-177)$ & $<0.001$ \\
IL-17 $(\mathrm{pg})$ & $37.4 \pm 8.8(27-58)^{\#}$ & $56.4 \pm 23.7(28-104)^{*}$ & $30.5 \pm 3.8(26-38)$ & $<0.001$ \\
IL-17 $(\mathrm{pg} / \mu \mathrm{L})$ & $57.4 \pm 34(23-164)^{* \#}$ & $42.2 \pm 10.5(28-67)^{* \#}$ & $84.1 \pm 34.9(42-148)$ & $<0.001$ \\
GCF $(\mu \mathrm{L})$ & $1.0 \pm 0.2(0.9-1.3)^{*}$ & $1.3 \pm 0.2(0.5-1.5)^{*}$ & $0.4 \pm 0.3(0.2-0.6)$ & $<0.001$ \\
\hline
\end{tabular}

GCP: generalized chronic periodontitis; GAgP: generalized aggressive periodontitis; C: control.

* Statistically significantly different from the $\mathrm{C}$ group.

"Statistically significantly different from the GAgP group.

TABLE 4: Ratios of the IL-11: IL-17 in GCF of CP, GAgP, and C subjects.

\begin{tabular}{lcccc}
\hline Cytokine ratio & GCP $(n=25)$ & GAgP $(n=25)$ & C $(n=15)$ & $\begin{array}{c}P \text { value from } \\
\text { Kruskall-Wallis } \\
\text { test }\end{array}$ \\
\hline IL-11: IL-17 & $1.46: 1^{*}$ & $1.09: 1^{*}$ & $1.62: 1$ & 0.011 \\
IL-11: IL-17 & $1.01: 1^{*}$ & $0.99: 1^{*}$ & $1.30: 1$ & 0.016 \\
\hline
\end{tabular}

GCP: generalized chronic periodontitis; GAgP: generalized aggressive periodontitis; C: control.

* Statistically significantly different from control group $(P<0.05)$.

${ }^{\text {a }}$ Ratio of the total amount of IL-11 to IL-17.

${ }^{b}$ Ratio of the concentration of IL-11 to IL-17.

lower IL-11 concentrations when compared to GCP group $(P<0.001)$. No significant differences were found among the groups in the total amount of IL-11 $(P=0.309)$.

3.3. Cytokine Ratios. The IL-11/IL-17 ratios, total amount, and concentration are shown in Table 4. The IL-11/IL-17 ratio was significantly higher in the $\mathrm{C}$ group than GAgP and GCP groups $(P<0.05)$. GAgP group showed lower ratios of IL-11/IL-17 when compared to GCP group; yet no statistically significant difference was observed between them.

3.4. Bacterial Detection by PCR. The occurrence of different periodontal bacteria, A. actinomycetemcomitans, T. forsythia, $T$. denticola, $P$. gingivalis, and $P$. intermedia, in the dental plaque of GCP and GAgP patients is given in Table 5. Detection frequency and percentage of $A$. actinomycetemcomitans were significantly higher in GAgP than in the GCP patients. Meanwhile, the detection frequency and percentage of $T$. denticola, $P$. gingivalis, and $P$. intermedia were significantly higher in GCP than in the GAgP patients. No statistically significant difference was detected in the frequency of $T$. forsythia between GAgP and GCP patients yet it was higher in GCP patients.

3.5. Relationship between Bacterial Status and GCF Cytokine Level. Figures 1 and 2 show the relationship between GCF levels of IL-17 and IL-11 and the occurrence of different bacteria (A. actinomycetemcomitans, T. forsythia, T. denticola, $P$. gingivalis, and P. intermedia) in the positive samples of GCP and GAgP patients. The high occurrence of P. gingivalis in the dental plaque was associated with significantly

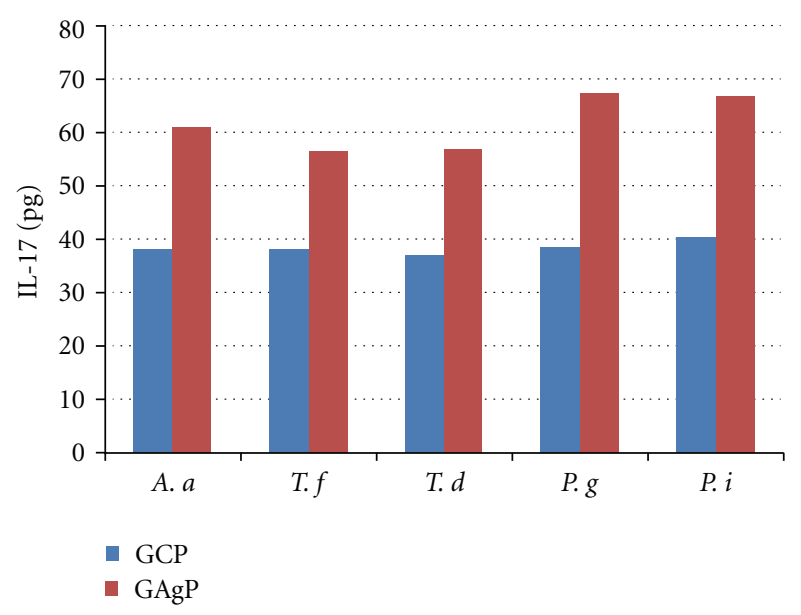

FIGURE 1: Mean GCF levels of total IL-17 based on the presence of different periodontal bacteria in the dental plaques of patients with GCP and GAgP.

increased GCF levels of IL-17 in GCP $(P<0.05)$ and GAgP patients $(P<0.001)$ in positive samples compared to negative samples. No significant differences in the GCF levels of the IL-11 depending on the presence of any periodontal bacteria were found (data not shown).

\section{Discussion}

The intensity, duration, and resolution of inflammation depend on shifting the balance between the activities of proinflammatory and anti-inflammatory cytokines during 
TABLE 5: The occurrence of different periodontopathic bacteria in the dental plaque of GCP and GAgP groups. Data represent the frequency and percentage of the individuals with given amount of periodontal pathogens in probes of dental plaque.

\begin{tabular}{lccr}
\hline & GCP $(n=25)$ & GAgP $(n=25)$ & $P$ value \\
\hline A. actinomycetemcomitans $\left(>10^{6}\right)$ & $8(32)$ & $11(44)$ & $<.001^{*}$ \\
T. forsythia $\left(>10^{6}\right)$ & $19(76)$ & $18(72)$ & $>0.05$ \\
T. denticola $\left(>10^{6}\right)$ & $18(72)$ & $13(52)$ & $<0.001^{*}$ \\
$P$. gingivalis $\left(>10^{6}\right)$ & $21(84)$ & $11(44)$ & $<0.001^{*}$ \\
$P$. intermedia $\left(>10^{6}\right)$ & $13(52)$ & $9(36)$ & $<0.001^{*}$ \\
\hline
\end{tabular}

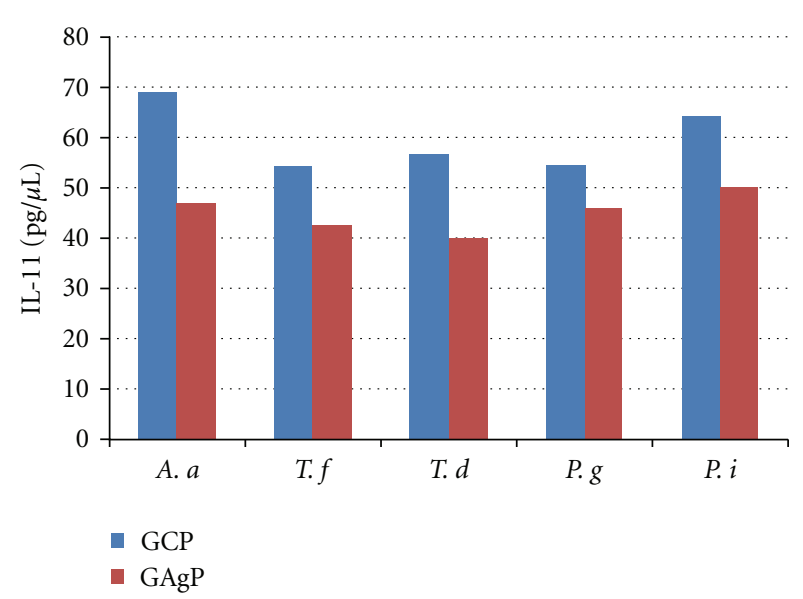

Figure 2: Mean GCF levels of IL-11 concentration based on the presence of different periodontal bacteria in the dental plaques of patients with GCP and GAgP.

the periodontal inflammation [21]. Accordingly, this investigation evaluated the total amount and concentration of IL-17 and IL-11 in GCF of GCP, GAgP, and C subjects.

The current study shows, for the first time, an overexpression in the total amount of IL-17 in GCF of GAgP patients compared to GCP and C ones. This might suggest a potential cellular hyperactivity that may favor periodontal destruction in GAgP. Recently, Duarte et al. [17] demonstrated that subjects with GAgP presented higher serum levels of IL17 than subjects with GCP and C, and that this increase was related to gene polymorphisms coding for the synthesis of inflammatory mediators. Likewise, Schenkein et al. [22] detected IL-17 in sera from GAgP patients, suggesting that it could be from the locally produced cytokine in the periodontal tissues. GAgP patients are known to exhibit defective neutrophils chemotactic responses and enhanced oxidative metabolic responses [23]. It has been assumed that elevated IL-17 levels noted in GAgP may represent a compensatory increase in cytokine production in response to these functional defects [22]. Conversely, Borch et al. [24] found no differences between C and GAgP subjects in the release of IL-17 by mononuclear cells, yet results from cell-culture methodologies are not directly comparable with cytokines GCF levels.

This study also showed comparable results to Vernal et al. [12] who reported significantly higher total amounts of IL-17 in GCF and in the culture supernatants of GCP patients than in C. Elevated tissue concentrations of IL-17 could promote periodontitis progression by increasing concentrations of bone resorbing chemokines, which suggests that IL-17 may be a mediator of periodontal destruction $[12,25]$. Previous studies showed that IL-17 induced the expression of prostaglandin $\mathrm{E}_{2}\left(\mathrm{PGE}_{2}\right)$ [26]. Others demonstrated that IL-17 has a synergy with TNF- $\alpha$ in synthesizing IL- 6 and increasing bone resorption [27]. Interestingly, IL-17 has been observed to act on osteoblasts inducing their differentiation into mature osteoclasts $[28,29]$.

Alternatively, this study demonstrated a significant decrease in IL-17 GCF concentration in patients with GCP and GAgP compared with $\mathrm{C}$, and in GAgP patients when compared with GCP patients. These results were supported by Vernal et al. [12] who reported lower IL-17 GCF concentration in GCP patients compared to $\mathrm{C}$ and attributed this decrease to the higher GCF volume produced in diseased sites with $\mathrm{PD}>5 \mathrm{~mm}$ than in healthy sites from $\mathrm{C}$.

Johnson et al. [31] reported lower IL-11 concentrations within gingiva adjacent to deep PD, suggesting an imbalance between pro- and anti-inflammatory mediators in their study. This investigation also observed significantly lower IL-11 concentrations in GAgP group which had the deepest PD and CAL in sampling sites, compared with GCP and $\mathrm{C}$ groups. Furthermore, GCP group had significantly decreased concentration of IL-11 than C group, which was in accordance with Yetkin et al. [30] and Johnson et al. [31] results. IL-11 has been shown to inhibit IL- $1 \beta$, TNF- $\alpha$, IL6 , and downregulated LPS-induced cytokines throughout the inhibition of NF- $\kappa \mathrm{B}$ expression in vitro [32]. Previous investigators demonstrated that IL-11 induced osteoblastic differentiation and bone formation in vivo and in vitro [33]. It has been reported that the twice-weekly administration of recombinant human IL-11 in periodontal disease model acted by blocking proinflammatory cytokines, leading to a reduction in attachment loss and bone resorption and improvement of the inflammatory reaction [14]. Taken together, the results mentioned above allow us to suggest that IL-11 might be a key mediator in preventing the progressive inflammation leading to periodontal tissue breakdown [30].

In this study the total amount and concentration of IL-11: IL-17 cytokine ratio was significantly higher in C group compared to both GCP and GAgP groups, and higher in GCP group compared to GAgP, that is, decreased with increasing PD. Johonson et al. [31] reported that IL11 : IL-17 ratios decreased from $3 \mathrm{~mm}$ to $>6 \mathrm{~mm}$ in diseased gingiva. Moreover, Yetkin et al. [30] showed higher IL$11:$ IL-17 ratio in healthy sulcus of C subjects compared 
to deep pockets of GCP patients suggesting that the antiinflammatory properties of IL-11 may be impaired in deeper pockets. Yücel et al. [16] also found significantly higher IL11 : IL- $1 \beta$ ratio in gingivitis and $\mathrm{C}$ groups compared to GCP group.

Recently, Andrukhov et al. [34] stated that due to differences in the bacterial load different types of periodontal diseases could be associated with specific cytokine profiles. It has been suggested that the plaque bacterial load could determine the type of periodontal disease; that is, A. actinomycetemcomitans was associated with GAgP and GCP was associated with the red-complex bacteria [35]. In this study, samples with positive $P$. gingivalis in GAgP and GCP patients showed significantly higher total amounts of IL-17 than negative samples. This agrees with another study where $P$. gingivalis induced a significant increase in IL-17 in periodontitis patients than in gingivitis patients [36]. Considering that IL-17 is capable of inducing the production of TNF- $\alpha$ [37], our findings are also supported by Andrukhov et al. [34] who reported that TNF- $\alpha$ serum levels were significantly increased in periodontitis patients with high plaque load of $P$. gingivalis.

In conclusion, the current findings suggested a role for IL-17 in the periodontal destruction of GAgP and GCP along with a therapeutic role for IL-11. Further investigations with larger populations are required to clarify the specific contribution of IL-17 to the pathogenesis of periodontitis and to determine the therapeutic benefit of IL-11 for resolution of inflammation in periodontal diseases.

\section{Conflict of Interests}

The authors declare that they have no conflict of interests.

\section{Acknowledgments}

The authors would like to thank Dr. Khaled Keraa, for his help in the statistical analysis of this work. The current study was funded by personal resources to be refunded later by the Ministry of Higher Education, Cairo, Egypt, on international publishing.

\section{References}

[1] G. C. Armitage, "Development of a classification system for periodontal diseases and conditions," Annals of Periodontology, vol. 4, no. 1, pp. 1-6, 1999.

[2] A. D. Haffajee and S. S. Socransky, "Microbial etiological agents of destructive periodontal diseases," Periodontology 2000, vol. 5, pp. 78-111, 1994.

[3] S. S. Socransky, A. D. Haffajee, M. A. Cugini, C. Smith, and R. L. Kent Jr., "Microbial complexes in subgingival plaque," Journal of Clinical Periodontology, vol. 25, no. 2, pp. 134-144, 1998.

[4] S. M. Opal and V. A. DePalo, "Anti-inflammatory cytokines," Chest, vol. 117, no. 4, pp. 1162-1172, 2000.

[5] G. E. Salvi and N. P. Lang, "Host response modulation in the management of periodontal diseases," Journal of Clinical Periodontology, vol. 32, no. 6, pp. 108-129, 2005.
[6] I. Ishikawa, K. Nakashima, T. Koseki et al., "Induction of the immune response to periodontopathic bacteria and its role in the pathogenesis of periodontitis," Periodontology 2000, vol. 14, pp. 79-111, 1997.

[7] C. T. Weaver, L. E. Harrington, P. R. Mangan, M. Gavrieli, and K. M. Murphy, "Th17: an effector CD4 T cell lineage with regulatory T cell ties," Immunity, vol. 24, no. 6, pp. 677-688, 2006.

[8] S. L. Gaffen and G. Hajishengallis, "A new inflammatory cytokine on the block: re-thinking periodontal disease and the Th1/Th2 paradigm in the context of Th17 cells and IL-17," Journal of Dental Research, vol. 87, no. 9, pp. 817-828, 2008.

[9] A. Beklen, M. Ainola, M. Hukkanen, C. Gürgan, T. Sorsa, and Y. T. Konttinen, "MMPs, IL-1, and TNF are regulated by IL-17 in periodontitis," Journal of Dental Research, vol. 86, no. 4, pp. 347-351, 2007.

[10] M. J. Ruddy, F. Shen, J. B. Smith, A. Sharma, and S. L. Gaffen, "Interleukin-17 regulates expression of the CXC chemokine LIX/CXCL5 in osteoblasts: implications for inflammation and neutrophil recruitment," Journal of Leukocyte Biology, vol. 76, no. 1, pp. 135-144, 2004.

[11] K. Takahashi, T. Azuma, H. Motohira, D. F. Kinane, and S. Kitetsu, "The potential role of interleukin-17 in the immunopathology of periodontal disease," Journal of Clinical Periodontology, vol. 32, no. 4, pp. 369-374, 2005.

[12] R. Vernal, N. Dutzan, A. Chaparro, J. Puente, M. A. Valenzuela, and J. Gamonal, "Levels of interleukin-17 in gingival crevicular fluid and in supernatants of cellular cultures of gingival tissue from patients with chronic periodontitis," Journal of Clinical Periodontology, vol. 32, no. 4, pp. 383-389, 2005.

[13] H. Ohyama, N. Kato-Kogoe, A. Kuhara et al., "The involvement of IL-23 and the Th 17 pathway in periodontitis," Journal of Dental Research, vol. 88, no. 7, pp. 633-638, 2009.

[14] G. Martuscelli, J. P. Fiorellini, C. C. Crohin, and T. H. Howell, "The effect of interleukin-11 on the progression of ligatureinduced periodontal disease in the beagle dog," Journal of Periodontology, vol. 71, no. 4, pp. 573-578, 2000.

[15] D. F. Kinane and R. Attström, "Advances in the pathogenesis of periodontitiss: group B consensus report of the fifth European workshop in periodontology," Journal of Clinical Periodontology, vol. 32, no. 6, pp. 130-131, 2005.

[16] O. O. Yücel, E. Berker, S. Gariboğlu, and H. Otlu, "Interleukin11, interleukin-1beta, interleukin-12 and the pathogenesis of inflammatory periodontal diseases," Journal of Clinical Periodontology, vol. 35, no. 5, pp. 365-370, 2008.

[17] P. M. Duarte, M. da Rocha, E. Sampaio et al., "Serum levels of cytokines in subjects with generalized chronic and aggressive periodontitis before and after non-surgical periodontal therapy: a pilot study," Journal of Periodontology, vol. 81, no. 7, pp. 1056-1063, 2010.

[18] M. Faveri, L. C. Figueiredo, P. M. Duarte, M. J. Mestnik, M. P. A. Mayer, and M. Feres, "Microbiological profile of untreated subjects with localized aggressive periodontitis," Journal of Clinical Periodontology, vol. 36, no. 9, pp. 739-749, 2009.

[19] J. Silness and H. Löe, "Periodontal disease in pregnancy. II. Correlation between oral hygiene and periodontal condition," Acta Odontologica Scandinavica, vol. 22, pp. 121-135, 1964.

[20] S. A. Syed and W. J. Loesche, "Survival of human dental plaque flora in various transport media," Applied Microbiology, vol. 24, no. 4, pp. 638-644, 1972.

[21] T. Honda, H. Domon, T. Okui, K. Kajita, R. Amanuma, and K. Yamazaki, "Balance of inflammatory response in stable gingivitis and progressive periodontitis lesions," Clinical and Experimental Immunology, vol. 144, no. 1, pp. 35-40, 2006. 
[22] H. A. Schenkein, T. E. Koertge, C. N. Brooks, R. Sabatini, D. E. Purkall, and J. G. Tew, "IL-17 in sera from patients with aggressive periodontitis," Journal of Dental Research, vol. 89, no. 9, pp. 943-947, 2010.

[23] M. I. Ryder, "Comparison of neutrophil functions in aggressive and chronic periodontitis," Periodontology 2000, vol. 53, no. 1, pp. 124-137, 2010.

[24] T. S. Borch, M. Løbner, K. Bendtzen, P. Holmstrup, and C. H. Nielsen, "Decreased interleukin-2 responses to fusobacterium nucleatum and porphyromonas gingivalis in generalized aggressive periodontitis," Journal of Periodontology, vol. 80, no. 5, pp. 800-807, 2009.

[25] S. R. Lester, J. L. Bain, R. B. Johnson, and F. G. Serio, "Gingival concentrations of interleukin-23 and -17 at healthy sites and at sites of clinical attachment loss," Journal of Periodontology, vol. 78, no. 8, pp. 1545-1550, 2007.

[26] F. Fossiez, O. Djossou, P. Chomarat et al., "T cell interleukin17 induces stromal cells to produce proinflammatory and hematopoietic cytokines," Journal of Experimental Medicine, vol. 183, no. 6, pp. 2593-2603, 1996.

[27] R. L. Van Bezooijen, S. E. Papapoulos, and C. W. G. M. Löwik, "Effect of interleukin-17 on nitric oxide production and osteoclastic bone resorption: is there dependency on nuclear factor- $\kappa \mathrm{B}$ and receptor activator of nuclear factor $\kappa \mathrm{B}$ (RANK)/RANK ligand signaling?” Bone, vol. 28, no. 4, pp. 378-386, 2001.

[28] T. Nakashima, Y. Kobayashi, S. Yamasaki et al., "Protein expression and functional difference of membrane-bound and soluble receptor activator of NF- $\kappa \mathrm{B}$ ligand: modulation of the expression by osteotropic factors and cytokines," Biochemical and Biophysical Research Communications, vol. 275, no. 3, pp. 768-775, 2000.

[29] E. Lubberts, L. van den Bersselaar, B. Oppers-Walgreen et al., "IL-17 promotes bone erosion in murine collagen-induced arthritis through loss of the receptor activator of NF- $\kappa \mathrm{B}$ ligand/osteoprotegerin balance1," Journal of Immunology, vol. 170, no. 5, pp. 2655-2662, 2003.

[30] Z. Yetkin Ay, R. Sütçü, E. Uskun, F. Y. Bozkurt, and E. Berker, "The impact of the IL-11:IL-17 ratio on the chronic periodontitis pathogenesis: a preliminary report," Oral Diseases, vol. 15, no. 1, pp. 93-99, 2009.

[31] R. B. Johnson, N. Wood, and F. G. Serio, "Interleukin-11 and IL-17 and the pathogenesis of periodontal disease," Journal of Periodontology, vol. 75, no. 1, pp. 37-43, 2004.

[32] Y. Takeuchi, S. Watanabe, G. Ishii et al., "Interleukin-11 as a stimulatory factor for bone formation prevents bone loss with advancing age in mice," The Journal of Biological Chemistry, vol. 277, no. 50, pp. 49011-49018, 2002.

[33] K. Suga, M. Saitoh, S. Kokubo et al., "Synergism between interleukin-11 and bone morphogenetic protein-2 in the healing of segmental bone defects in a rabbit model," Journal of Interferon and Cytokine Research, vol. 24, no. 6, pp. 343-349, 2004.

[34] O. Andrukhov, C. Ulm, H. Reischl, P. Q. Nguyen, M. Matejka, and X. X. Rausch-Fan, "Serum cytokine levels in periodontitis patients in relation to the bacterial load," Journal of Periodontology, vol. 82, no. 6, pp. 885-892, 2011.

[35] G. C. Armitage, "Comparison of the microbiological features of chronic and aggressive periodontitis," Periodontology 2000, vol. 53, no. 1, pp. 70-88, 2010.

[36] T. Oda, H. Yoshie, and K. Yamazaki, "Porphyromonas gingivalis antigen preferentially stimulates $\mathrm{T}$ cells to express IL17 but not receptor activator of NF- $\kappa \mathrm{B}$ ligand in vitro," Oral Microbiology and Immunology, vol. 18, no. 1, pp. 30-36, 2003.
[37] C. M. Eklund, "Proinflammatory cytokines in CRP baseline regulation," Advances in Clinical Chemistry, vol. 48, pp. 111136, 2009. 


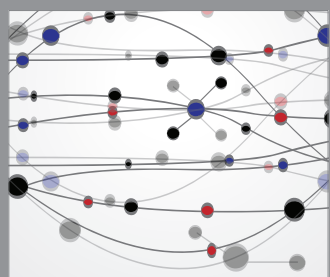

The Scientific World Journal
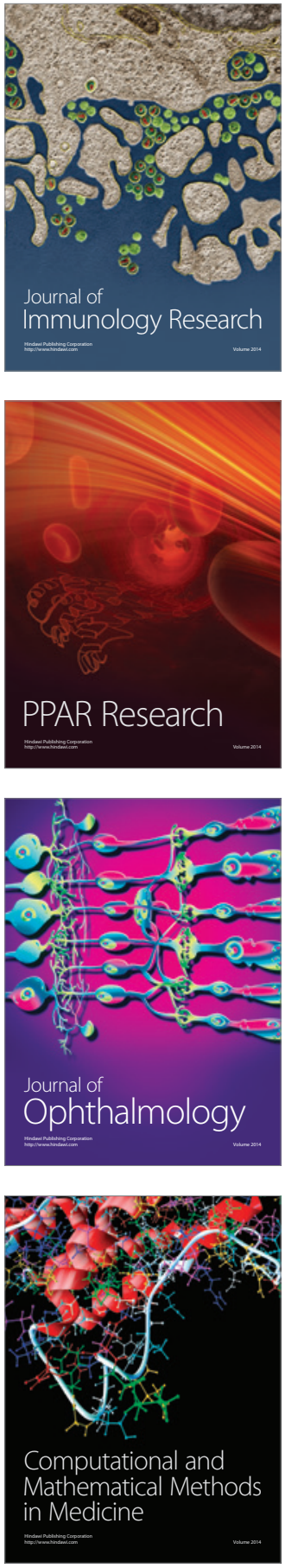

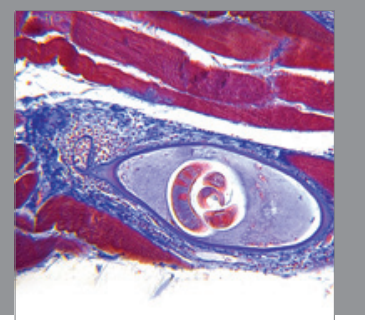

Gastroenterology

Research and Practice
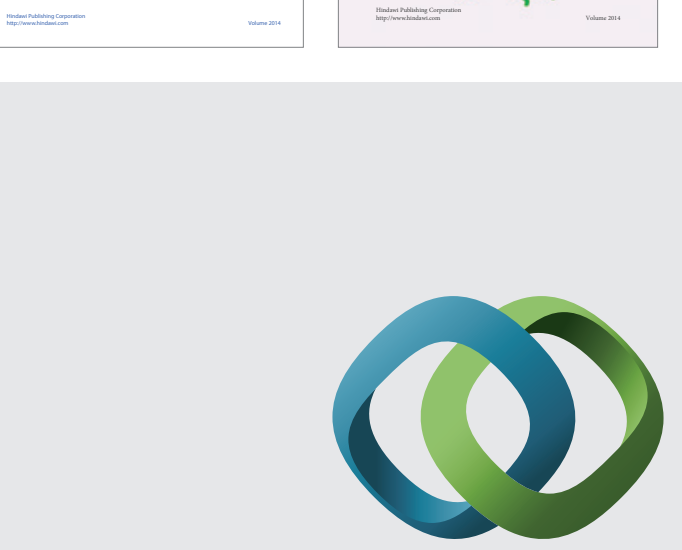

\section{Hindawi}

Submit your manuscripts at

http://www.hindawi.com
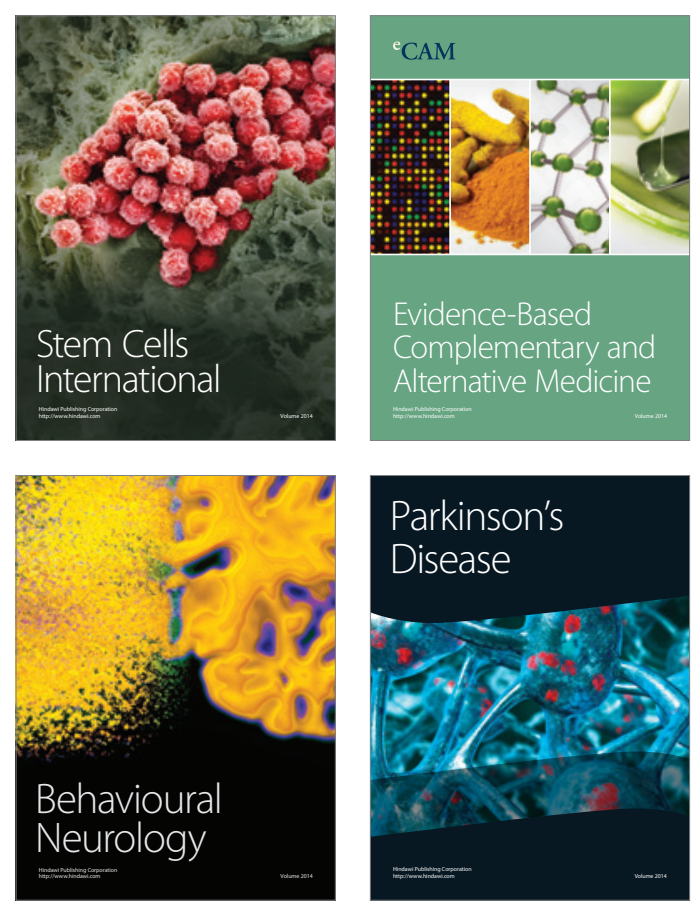

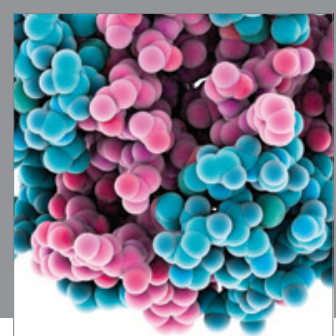

Journal of
Diabetes Research

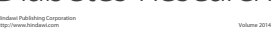

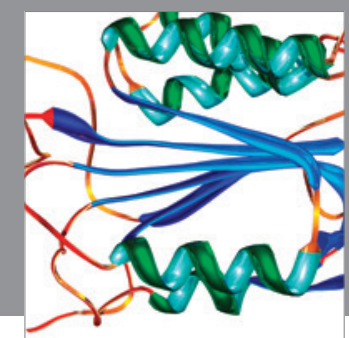

Disease Markers
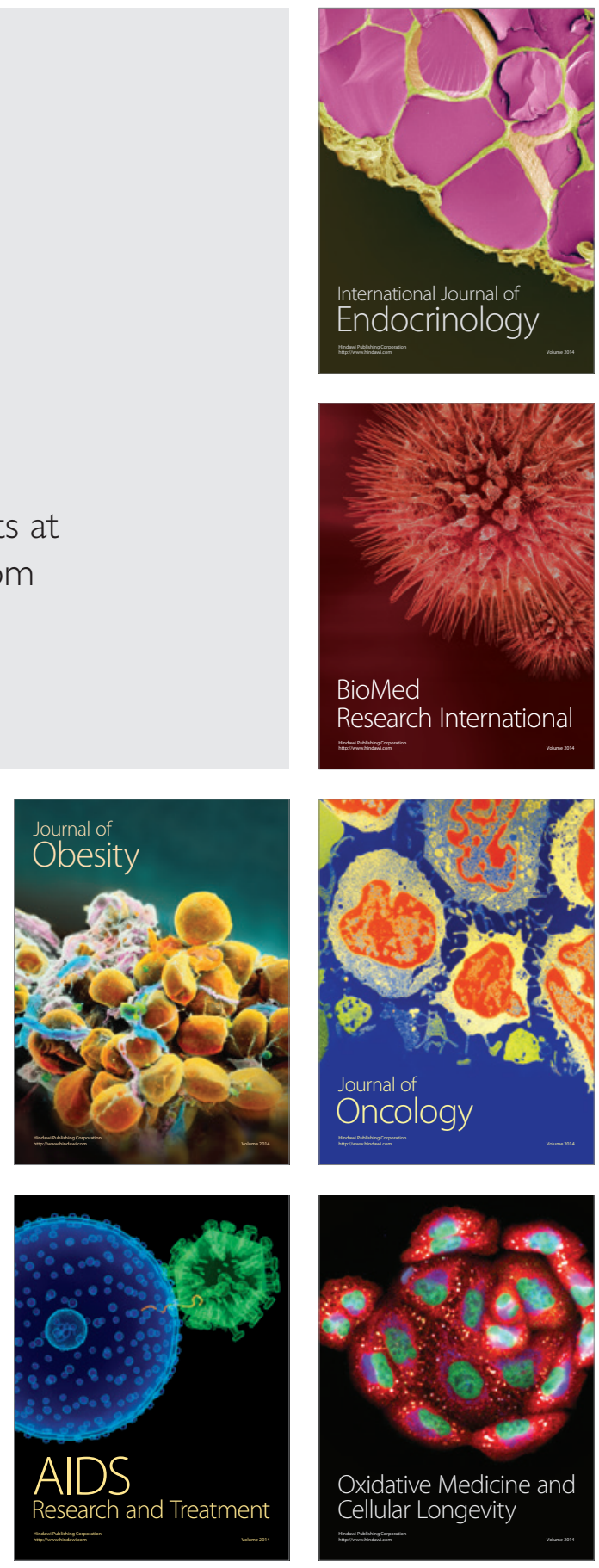